\title{
Das Unnütze Wissen (der Literaturwissenschaft)
}

Meine Titelformulierung vom Unnützen Wissen (der Literaturwissenschaft) lässt sich doppelt lesen. Einmal im Hinblick auf eine Unterscheidung innerhalb der Literaturwissenschaft selbst. Dann ginge es darum zu fragen, welches Wissen in und für die Literaturwissenschaft unnütz und welches nützlich sei. Zum Beispiel könnte man dann sagen, dass das Wissen um Verfahren der Edition nützlich, das Wissen über Unnützes Wissen der Literatur aber unnütz sei etc. Zum anderen aber lässt sich mein Titel auch lesen im Hinblick auf eine Unterscheidung im Wissen überhaupt. Dann ginge es darum zu fragen, ob etwa das Wissen der Physik oder der Medizin nützlich, das der Literaturwissenschaft aber unnütz sei. Denn das Wissen um rhetorische Figuren oder die literaturtheoretischen Debatten um Dekonstruktion und Diskursanalyse mag für den Literaturwissenschaftler in seiner Disziplin nützlich sein, aber was nützt dieses Wissen außerhalb ihrer selbst, welchen Nutzen stellt das Wissen der Disziplin Literaturwissenschaft der Gesellschaft zur Verfügung? Wollte man die Frage in dieser Weise stellen, dann ließe man sich allerdings immer schon auf die Kategorie der Nützlichkeit des Wissens ein. Man würde dann vielleicht argumetieren, dass die Literaturwissenschaft für die - auch in ökonomischen Zusammenhängen so nützliche Text- oder Lesekompetenz sorge und am Ende gar behaupten, sie sei nützlicher als etwa die Geschichtswissenschaft, deren Wissen über die Bestattungsriten der Neandertaler nun wirklich nutzlos wäre. Gerade dieses argumentative Wettrennen um die eigene disziplinäre Nützlichkeit soll aber hier unterbleiben - und eben deshalb steht die Literaturwissenschaft in meinem Titel in Klammern. Mehr als um das Unnütze Wissen der Literaturwissenschaft im Besonderen geht es im Folgenden um die Frage und die Geschichte des Unnützen Wissens überhaupt, und zwar ohne die Kategorie des Nützlichen selbst bereits zu akzeptieren. Vielmehr möchte ich als Literaturwissenschaftler fragen, wie und warum eine solche Kategorisierung des nützlichen und des unnützen Wissens entstanden ist und wie man ihr - mit diesem Wissen - vielleicht begegnen könnte.

Zunächst: Was ist Wissen? Ansetzen möchte ich bei der grammatischen Tatsache, dass es sich bei dem Verb swissen um ein präteritopräsentisches Verb handelt. ’Präteritopräsens` ist eine Bezeichnung für Vergangenheitsformen mit präsentischer Bedeutung. So beschreibt `Ich weiß das Ergebnis 
eines vergangenen Prozesses, bezogen auf das Subjekt, und heißt eigentlich: IIch habe gesehen ${ }^{1}$ Es geht also um einen Zustand als Folge eines abgeschlossenen Prozesses - ich weiß etwas erst, wenn ich es gesehen, erfahren oder gelernt habe. Das Verb rwissen die in der Gegenwart des Wissens präsent ist (oder auch wieder vergessen wird): Wissen ist in diesem genauen Sinne ein Präteritopräsens. Wenn nun etwas zu wissen auf die Kategorie der Nützlichkeit (in ihrer heutigen Bedeutung) bezogen wird, so bekommt das durch Vergangenheitsbezug gewonnene Wissen tendenziell über das Präsens des Wissens hinaus einen futurischen Bezug. Die Nützlichkeit dessen, was ich weiß, bemisst sich an seiner Potentialität für die Gegenwart und mehr noch für die Zukunft: Ich weiß etwas (habe etwas erfahren), das mir nützlich ist, indem es mich befähigt, zu handeln bzw. mich zu erhalten oder Ziele zu erreichen. Insofern spreizt sich im Begriff des nützlichen Wissens eine spezifische Temporalität auf zwischen erfahren, gelernt haben einerseits und handeln andererseits. Etwas Nützliches zu wissen heißt, dass ich als Subjekt in der Vergangenheit etwas erfahren habe, das jetzt und für die Zukunft wichtig sein wird.

Das Substantiv 'Wissen löst nun, wenn es in objektiver Bedeutung gebraucht wird, den subjektiven Aspekt des Präteritums rich habe erfahren<, rich habe gesehen tendenziell auf, indem es allein das Wissbare bezeichnet, die Menge all dessen, was gewusst werden kann, sozusagen unabhängig vom subjektiven Akt des Sehens oder Erfahrens. ${ }^{2}$ Die Belege für eine Substantivierung des Verbs ‘wissen` zur objektiven Bedeutung von >das Wissen häufen sich erst seit dem letzten Drittel des 18. Jahrhunderts. ${ }^{3}$ Diese Sub-

1 »Präteritopräsentia sind Verben, die der Form nach ein Präteritum, der Bedeutung nach aber ein Präsens darstellen. Ein Beispiel dafür ist das Verb wissen: Es leitet sich aus einer Wurzel ab, die ssehen bedeutet: was man gesehen hat, das weiß man." Elke Hentschel/ Petra M. Vogel (Hg.), De Gruyter-Lexikon, Deutsche Morphologie, Berlin 2009, S. 338.

2 Vgl. zur Diskussion eines objektiven bzw. »impersonalen« Begriffs des Wissens: Tilmann Köppe, Vom Wissen in Literatur, in: ZfG N.F. 17/2 (2007), S. 398-410, hier S. 406ff. Köppe bestreitet den Sinn des Begriffs von impersonalem Wissen und beharrt darauf, dass Wissen immer eine Beziehung zwischen Subjekt und Sachgehalt bezeichnet. Er unterschlägt damit den begriffsgeschichtlichen Prozess der Substantivierung und seinen medienhistorischen Hintergrund. Kritisch zu Köppes Bestreitung eines Begriffs von rimpersonalem Wissen Wissen in Literatur, in: ZfG N.F. 18/2 (2008), S. 373-377, hier S. 376.

3 In Zedlers Universal-Lexikon gibt es keinen Eintrag zum Substantiv Das Wissen. Bei Adelung gibt es zwar einen solchen Eintrag, aber zugleich den Hinweis, dass das Wort "doch nur selten" vorkomme. Adelung kennt noch den Begriff "Wissenheit", das aber "für sich allein veraltet" sei und nur noch in `Unwissenheit vorkomme; sowie das Wort 'Wissenschaft, das aber im Sinne von 'Ich habe keine Wissenschaft von der Sache im Hochdeutschen zu veralten" beginne. Johann Christoph Adelung, Grammatisch-kriti- 
stantivierung ermöglicht eine diskursive Objektivierung dessen, was potentiell gewusst werden kann: Es bezeichnet eine Datenmenge, die für subjektive Akte des Erfahrens/Sehens gleichsam bereitsteht - und die sich durch solche subjektive Akte und ihre Vezeichnung bzw. Weitergabe die ganze Zeit über ändert, erweitert oder auch verschiebt. 'Das Wissen< bezeichnet gleichsam den sich verändernden oder erweiternden, sich verschiebenden Pool aller möglichen diskursiv jeweils vorrätigen Erfahrungen und Kenntnisse. Damit löst sich das Substantiv zunächst von der spezifischen subjektiven Temporalität des präteritopräsentischen Verbs ab. Nicht sich weiß< (im Sinne von rich habe erfahren $)$, sondern: Es gibt das Wissen der Physiognomik, der Medizin, der Physik, der Literaturwissenschaft etc. Das Wissen, das als Singularetantum zugleich plurale Bedeutung hat, impliziert eine bestimmte Menge an Daten bzw. Informationen etc., die auf bestimmte Weise medial da sind und nun als medial gebundene ihrer eigenen zeitlichen Dynamik unterworfen sind. ${ }^{4}$ Die Substantivierung des Wortes 'Wissen $<$ korreliert so mit der aufklärerischen Reflexion von Medien und vor allem von Schrift als zentralem Speichermedium von Wissen sowie - damit zusammenhängend - mit dem Prozess der Verzeitlichung von Zeit, wie er sich etwa im Historismus Herders niederschlägt. ${ }^{5}$ Man kann >das Wissen $`$ nun reflexiv entweder auf Großsubjekte beziehen, wie das Wissen der Gesellschaft, das Wissen der heutigen Zeit, das Wissen vergangener Gegenwarten, das Wissen des Menschen etc. - oder aber den Bezug auf Subjekte des Wissens ganz beiseitelassen. Man kann dann einfach sagen, das Wissen ist genau oder ungenau, sicher oder unsicher, praktisch oder theoretisch, aktuell oder veraltet, mehr oder weniger, oder eben: nützlich oder unnütz.

Im Hinblick auf die Frage der Nützlichkeit des Wissens spreizt sich auch beim Substantiv eine spezifische Temporalität auf. Auch hier verknüpft sich

sches Wörterbuch der hochdeutschen Mundart, Bd. 4, Wien 1811, Sp. 1582. Die frühesten Grimm'schen Belege für sdas Wissen in objektiver Bedeutung stammen von Lavater, Herder und Goethe. Jacob und Wilhelm Grimm, Artikel Wissen, in: Diess., Wörterbuch der deutschen Sprache, Leipzig 1854, Bd. 30, Sp. 743-748.

4 "Als strukturierte Menge von Kenntnissen, die in kulturellen Systemen erworben und aktualisiert, modifiziert und weitergegeben werden, unterliegt Wissen einer zeitlichen Dynamik." Ralf Klausnitzer, Literatur und Wissen. Zugänge, Modelle, Analysen, Berlin/ New York 2008, S. 29. Ein zu einer bestimmten Zeit publiziertes Lexikon veraltet eben mit der Zeit.

5 »Tiefer gesehen, ist der Historismus die Ermöglichung dieser Differenz von gegenwärtiger Vergangenheit und vergangenen Gegenwarten, wurzelt also im Reflexivwerden der Zeit selbst.« Niklas Luhmann, Weltzeit und Systemgeschichte, in: Hans Michael Baumgartner/Jörn Rüsen (Hg.), Seminar: Geschichte und Theorie: Umrisse einer Historik, Frankfurt a.M. 1976, S. 354. 
die Vergangenheit der Wissenserhebung und der (medial und diskursiv bedingten) Wissensweitergabe und der (medial und diskursiv bedingten) Akkumulation von Wissen mit der Frage nach seiner jetzigen oder zukünftigen Anwendung. Beim Substantiv >Wissen und der Frage seiner Nützlichkeit geht es dabei aber um die Zeitlichkeit bzw. die Temporalstruktur der Gesellschaft selbst. Die Frage, wie Wissen und Nützlichkeit jeweils verkoppelt bzw. korreliert werden, hat seinen tieferen Grund in der jeweiligen Temporalstruktur der Gesellschaft und ihrer diskursiven Reflexivität. Dafür spricht nicht zuletzt, dass die Herausbildung des objektiv gebrauchten Substantivs >das Wissen und die Hochkonjunktur der gesellschaftstheoretischen Kategorie der 'Nützlichkeit bei Hume, Helvetius oder Bentham zeitlich zusammenfallen: in die zweite Hälfte des 18. Jahrhunderts, d.h. genau in die Zeit der »Historisierung der Zeit«. ${ }^{6}$

Anders gesagt, die Kategorie der Un/Nützlichkeit durchläuft (gerade in ihrer Anwendung auf das Wissen) seit der zweiten Hälfte des 18. Jahrhunderts selbst einen Transformationsprozess, der mit dem Prozess der Verzeitlichung der Zeit verzahnt ist. Das möchte ich anhand von Rousseaus Erziehungsroman Emil zeigen, der intensiv über das nützliche und das Unnütze Wissen nachdenkt und dabei seinerseits einen literarischen Text, nämlich einen Roman, ins Spiel bringt. Rousseaus Ausgangshypothese seines gesamten pädagogischen Unternehmens und der Beantwortung der Frage nach der Nützlichkeit dieses Unternehmens für den fiktiven Zögling Emil ist eine Reflexion der Zeit, die er als Medium permanenter Veränderung beschreibt:

Wenn der Mensch immer in seinem Lande verhaftet bliebe, wenn immer das gleiche Wetter herrschte, wenn niemand seinen Stand wechselte, so wäre die herrschende Praxis in gewisser Weise gut. Das Kind, einmal für seinen Beruf erzogen, brauchte ihn niemals mehr zu verlassen und wäre niemals den Unbequemlichkeiten eines anderen ausgesetzt. Aber die Verhältnisse ändern sich ständig, der Geist des Jahrhunderts ist unruhig und stürzt von Generation zu Generation alles um. Ist es daher nicht unsinnig, ein Kind so zu erziehen, als brauchte es sein Zimmer nie zu verlassen, als bliebe es immer inmitten seiner Leute? ${ }^{7}$

Derlei Reflexionen auf die Zeitlichkeit aller Bedingungen und Verhältnisse finden sich auch bei anderen Autoren der gleichen Zeit. Diderot etwa legt seiner Dramenreform die Einsicht zugrunde, dass, wie es heißt, »täglich

6 Ebd., S. 352.

7 Jean-Jacques Rousseau, Emil oder Über die Erziehung, in neuer dt. Fassung besorgt von Ludwig Schmidts, Paderborn/Wien/Zürich 1991, S. 15. 
neue Stände entstehen« ${ }^{8}$ In Lenz' Hofmeister belehrt der aufgeklärte Geheime Rat von Berg seinen Bruder: „Unsere Kinder sollen und müssen das nicht werden, was wir waren: die Zeiten ändern sich, Sitten, Umstände, alles. ${ }^{9}$ Und bei Thomas Paine kann man lesen: »The circumstances of the world are continually changing, and the opinions of men change also. ${ }^{10}$

Rousseau nun entwickelt vor diesem Hintergrund ein Modell von Erziehung, das angesichts dieser permanenten Veränderung eine für den Erwerb von Wissen und Erfahrung spezialisierte Eigenzeit einrichtet, die auf eine spätere Zeit bezogen ist. Die wichtigste und nützlichste Regel jeder Erziehung lautet hierbei: "nicht: Zeit gewinnen, sondern Zeit verlieren. ${ }^{11}$ Das Prinzip ist, für die Kindheit Zeit zu opfern, die man dann aber mit Zinsen zurückerhält. Gefordert wird ein vor den Anforderungen der Gegenwart geschützter Zeitraum für die Kindheit, der auf die sich wandelnde Zeit der Gesellschaft, auf zukünftige Gegenwarten bezogen ist. ${ }^{12}$ Das Ziel ist die Ausbildung eines "Menschen an sich ${ }^{13}$ der unter sich wandelnden Bedingungen anpassungs- und lernfähig bleibt. Der Mensch soll nicht für einen bestimmten Platz, Stand oder Beruf erzogen werden, sondern seine Berufung ist, Mensch zu sein, das "Leben ist sein Beruf: ${ }^{14}{ }^{2}$ Und wenn das Schicksal ihn zwingt, seinen Platz zu wechseln, er wird immer an seinem Platz sein. ${ }^{15}$

Diese Erziehung für unvorhersehbare zukünftige Gegenwarten arbeitet nun selbst mit dem Prinzip des nützlichen Wissens, das innerhalb dieser geschützten Eigenzeit ganz auf die Gegenwart und die Perspektive des Kindes bezogen bleiben kann. "Ein Erwachsener muß vieles wissen, dessen Nutzen

8 Denis Diderot, Unterredungen über den `Natürlichen Sohn<, in: Ders./Lessing, Das Theater des Herrn Diderot. Aus dem Französischen übersetzt von Gotthold Ephraim Lessing. Anmerkungen und Nachwort von Klaus-Detlef Müller, Stuttgart 1986, S. 159.

9 Jakob Michael Reinhold Lenz, Der Hofmeister, in: Ders., Werke und Briefe, hg. von Sigrid Damm, Bd. 1: Dramen, Frankfurt a.M./Leipzig 1987, S. 43.

10 Thomas Paine, Rights of man (1791), in: Ders., The Writings of Thomas Paine, hg. von Moncure Daniel Conway, Bd. 2, New York 1967, S. 281. Vgl. auch Paul Henri Thiry d'Holbach, System der Natur oder von den Gesetzen der physischen und der moralischen Welt, übers. von Fritz-Georg Voigt, Frankfurt a.M. 1978, S. 80: "Ändert sich nicht alles um uns herum? Ändern wir uns nicht selbst?《

11 Jean-Jacques Rousseau, Emil, S. 72.

12 Noch einmal Luhmann über das neuzeitliche Zeitbewusstsein: „Die Gegenwart versteht sich als Vergangenheit künftig-kontingenter Gegenwarten und wählt sich selbst als Vor-Auswahl künftiger Kontingenz. [...] Deshalb wird Zukunft als Horizont von Selektivität thematisch.« Niklas Luhmann, Weltzeit, S. 369f.

13 Jean-Jacques Rousseau, Emil, S. 15.

14 Ebd., S. 14 [Hervorh. JL].

15 Ebd. 
ein Kind noch nicht einsehen kann. ${ }^{16}$ Daher sei dem Kind solches Wissen als für es nutzlos auch zu ersparen. Vielmehr soll das Kind lernen, "was seinem Alter nützlich ist « ${ }^{17}$ und nichts weiter. "Aber«, so fährt Rousseau den möglichen Einwand selbst formulierend fort, "wird dann noch Zeit sein, das zu lernen, was man wissen muß, wenn der Augenblick gekommen ist, sein Wissen auch anzuwenden? Ich weiß es nicht. Was ich aber weiß, ist, daß es unmöglich ist, es früher zu lernen. ${ }^{18}$ Was das Kind allerdings schon jetzt, im Alter, in dem es prinzipiell den Nutzen der Voraussicht erkennen kann, lernen soll, ist die Kategorie der Nützlichkeit des Wissens selbst, es soll lernen, »nur Nützliches wissen zu wollen«. ${ }^{19}$ Rousseau schreibt: »Wozu nützt das? Das ist von nun an das geheiligte Wort, das zwischen ihm und mir über alles Tun in unserem Leben entscheidet. $\aleph^{20}$ Mit der Frage: »Wozu nützt das? « kann der Erzieher nun zum einen die Wissbegierde des Zöglings lenken, sieht sich aber andererseits auch selbst der Frage ausgesetzt, die von nun an der Zögling auch an den Erzieher richten wird. Was dabei herauskommt, ist ein Trainingslager des nützlichen Wissens in der jeweiligen Gegenwart, d.h. das Lernen der Zukunfts- und Voraussichtskategorie der Nützlichkeit aus der konkreten kindlichen Gegenwart heraus. Und genau um dieser Gegenwart willen soll man Zeit verlieren wollen, d.h. sich von der Nützlichkeit für die jetzige Gegenwart der Erwachsenen abkoppeln. In dieser eigens eingerichteten Eigenzeit sollen Wissen und Nutzen möglichst eng - durch Erfahrung in der Gegenwart - miteinander verknüpft werden, damit Emil in der unvorhersehbaren zukünftigen Gegenwart jeweils das nützliche Wissen selbst suchen und finden können wird. Dass dazu dann noch Zeit sein wird, so dass man sich jetzt leisten kann, um dieser zukünftigen Zeit willen Zeit zu verlieren, ist der Zeit- und Zukunftsoptimismus, der Rousseaus Zurichtung und Instrumentalisierung der kindlichen Neugier für die Fokussierung auf das Nützliche zugrunde liegt. Das Paradox besteht

${ }^{16}$ Ebd., S. 173.

17 Ebd.

18 Ebd. Diese zeittheoretische Grundlegung der Pädagogik nimmt später Schleiermacher auf: "Jede pädagogische Einwirkung stellt sich dar als Aufopferung eines bestimmten Moments für einen zukünftigen, und es fragt sich, ob wir befugt sind, solche Aufopferungen zu machen? « Friedrich Ernst Daniel Schleiermacher, Theorie der Erziehung. Die Vorlesungen aus dem Jahr 1826, Nachschriften, in: Ders., Ausgewählte pädagogische Schriften. Besorgt von Ernst Lichtenstein. Paderborn 1959, S. 36-243, hier S. 82. Siehe hierzu auch Manfred Lüders, Zeit, Subjektivität und Bildung. Die Bedeutung des Zeitbegriffs für die Pädagogik, Weinheim 1995, S. 113.

19 Jean-Jacques Rousseau, Emil, S. 173.

20 Ebd. 
demnach darin, dass einerseits der Nutzen eine Kategorie ist, die mit der Voraussicht auf die Zukunft gerichtet ist, die aber als Gegenwartserfahrung etabliert werden muss, und dass hierfür in einer sich wandelnden Gesellschaft selbst eigens Zeit zur Verfügung gestellt werden muss.

Rousseaus Beispiel für die Erfahrung des nützlichen Wissens ist gleichsam das Paradigma des nützlichen Wissens, nämlich Orientierungswissen im Moment der Gefahr. Während Jean-Jacques mit seinem Schüler »den Lauf der Sonne und die Art studier[t] e, wie man sich orientiert «, ${ }^{21}$ fragt ihn Emil, wozu das nütze sein soll. Dieselbe Frage stellt Emil angesichts der geographischen Belehrung über die unmittelbare Umgebung (dass der Wald nördlich von Montmorency liegt). Anstelle eines Vortrags hierüber inszeniert Jean-Jacques am nächsten Tag eine Situation, in der er und Emil sich in genau diesem Wald verirren, so dass das Wissen der Astronomie und der Geographie, wie mit dem Sonnenlauf und der Kenntnis von Himmelsrichtungen und geographischen Verhältnissen der Weg nach Hause gefunden werden kann, als nützliches Wissen unmittelbar mit der Bewältigung dieser konkreten Gefahr, als Verirrte im Wald Hunger zu leiden, verbunden wird. Jean-Jacques reduziert so die Kategorie des Nutzens auf erfahrbares, gegenwärtiges und letztlich körperbezogenes Handlungswissen; auf, so könnte man sagen, Rettungswissen. Schließlich will Emil vor allem deshalb aus dem Wald herausfinden, weil er schon großen Hunger hat. Nutzen wird zurückgebunden an den Körper und an Handlungen, die sich auf ihn und seine Erhaltung beziehen. Am plausibelsten ist die Nützlichkeit des Wissens offenbar im Moment der Gefahr, im Moment der Bedrohung, nämlich als Rettungswissen, da in der Situation der Gefahr das gegenwärtige Moment und das zukünftige - in der vollbrachten Selbsterhaltung - zusammenfallen. Nichts macht die Nützlichkeit des Wissens so evident wie Situationen, in denen das Wissen Mittel der Rettung bzw. des Überlebens ist. Das Paradigma für den ausschließlichen Bezug auf nützliches Wissen ist für Rousseau daher folgerichtig Daniel Defoes Robinson Crusoe, das einzige Buch, das für lange Zeit Emils "ganze Bibliothek « ${ }^{22}$ ausmacht, das er allerdings nur in gekürzter Form lesen darf: „Dieser Roman muß von seinem überflüssigen Beiwerk befreit werden. Er muß mit dem Schiffbruch Robinsons beginnen und mit der Ankunft des rettenden Schiffes enden. ${ }^{23}$ Ausgespart werden muss die Vorgeschichte des Vater-Sohn-Konflikts, die Robinson als seine

21 Ebd., S. 175.

22 Ebd., S. 180.

23 Ebd., S. 180 f. 
Ursünde beschäftigt, sowie sein Reichtum am Ende, der gleichsam als Belohnung seiner Bußzeit auf der Insel erscheint. ${ }^{24}$ Ins Bewusstsein des jungen Lesers soll allein jener Crusoe treten, der die ganze Zeit mit den drängenden Gegenwartsbedürfnissen kämpft und bei den Versuchen, sie zu befriedigen, unmittelbar merkt, welches Wissen nützlich ist und welches nicht. Der gerettete Schiffbrüchige auf einer einsamen Insel ist somit in einer für den Test auf die Nützlichkeit des Wissens idealen Situation, da er all sein Wissen unmittelbar für seine Selbsterhaltung einsetzen muss und dabei auf sich ganz allein gestellt ist - das nützliche Wissen also auch selbst erzeugen bzw. finden muss. Das Kind wird sich, so Rousseau, begeistert in Robinson Crusoe versetzen. »Es möchte alles wissen, was nützlich ist. Aber auch nur das! ${ }^{25}$ Das nützliche Wissen schlechthin ist das rettende Wissen, das Wissen zum Überleben. Und die Nützlichkeit dieses rettenden Wissens erweist sich immer nur innerhalb einer bestimmten Geschichte, in der im konkreten Ablauf der Zeit eine Krise eintritt, die durch ein Wissen im gegenwärtigen Moment der Gefahr gelöst wird - und erst im Moment dieser Gefahr bzw. der Rettung erweist sich, welches Wissen tatsächlich nützlich gewesen sein wird und welches nicht. Gerade wenn man - angesichts einer sich permanent wandelnden Welt - nicht voraussehen kann, welches Wissen in Zukunft nützlich sein wird, muss man das Finden des Nützlichen in der je-

24 Ausgespart werden muss auch der noch im gleichen Jahr (1719) erschienene gesamte zweite Teil, mit dem inhaltsangebenden Titel: "The Farther Adventures of Robinson Crusoe; Being the Second and Last Part of His Life, And of the Strange Surprising Accounts of his Travels Round three Parts of the Globe", der in den Ausgaben des 18. Jahrhunderts oft dem ersten Teil gleich beigefügt war. Für diesen Hinweis danke ich Roland Borgards.

25 Ebd., S. 181. Robinson Crusoe als Trainingsmedium nützlichen Wissens steht auch im Zentrum von Johann Heinrich Campe, Robinson der Jüngere, zur angenehmen und nützlichen Unterhaltung für Kinder. Nach dem Erstdruck hg. von Alwin Binder/Heinrich Richartz, Stuttgart 2005 (zuerst Hamburg 1779). Campe zitiert in der Vorrede die Passage aus Rousseaus Emil über Robinson Crusoe und kommt in seiner Version immer wieder auf die Kategorie des nützlichen Wissens und die Nützlichkeit der Not zurück. Diese besteht darin, dass er "sich immer selbst fragte: wozu mögte das wohl nützlich sein?« Ebd., S. 94. Allerdings geht es hier nicht allein um den Nutzen des Wissens für das eigene Überleben, sondern mehr noch um ein Training der Theodizee: Alle Ereignisse sind als weise Veranstaltungen Gottes zu bewerten, die für die eigene moralische Vervollkommnung nützlich sind. Johann Karl Wezels Robinson Krusoe, der 1779 und 1780 in zwei Bänden erschien, fokussiert dagegen weder das nützliche Wissen noch die Theodizee, sondern die Nutzlosigkeit historischer Erfahrung angesichts eines durch Egoismus, Herrschaftssucht und Besitzgier angetriebenen 'Fortschritts<. Vgl. hierzu Jörg Schönert, Wezels und Campes Bearbeitung des 'Robinson Crusoer. Zur literarischen Durchsetzung des bürgerlichen Wertkomplexes >Arbeit in der Literatur des späten 18. Jahrhunderts, in: Ders., Perspektiven zur Sozialgeschichte der Literatur. Beiträge zu Theorie und Praxis, Tübingen 2007, S. $97-112$. 
weiligen Gegenwart trainieren - als Fähigkeit, die dann auch für zukünftige Gegenwarten bereitsteht.

Rousseau hatte also eine Eigenzeit für die Erziehung eingerichtet, die jenseits der unmittelbaren ökonomischen Nützlichkeit genutzt werden soll, um die Kategorie der Nützlichkeit und des nützlichen Wissens in der Gegenwart (und zugleich freigestellt von ihr) für die Zukunft zu trainieren. Die Gründung der Humboldt-Universität um 1810 betreibt analog hierzu ebenfalls die Einrichtung einer Eigenzeit, in der die studierende Jugend von der Verpflichtung auf Fragen der unmittelbaren Nützlichkeit für die Gegenwart freigestellt wird. Schon Friedrich Schiller plädiert in seiner Jenaer Antrittsvorlesung dafür, in diesem Sinne Zeit zu verlieren. Während der philosophische Kopf gerade dasjenige Wissen sucht, »das den Geist nur als Geist vergnügen kann«, so lässt der Brotgelehrte nur solches Wissen als nützlich gelten, das er in seinem Amt brauchen kann. »Alle Zeit«, so Schiller weiter, die der Brotgelehrte dem bloß geistig vergnüglichen Wissen widmet, "würde er seinem künftigen Berufe zu entziehen glauben und sich diesen Raub nie vergeben. $\ll^{26}$ Ähnlich argumentiert Henrik Steffens in seinen Vorlesungen Über die Idee der Universitäten (1809). Auch hier geht es darum, dass die Studierenden in der Institution Universität aus »dem Drang des gegenwärtigen Lebens", dem der Staat unterliegt, »heraustreten« sollen, um eine Zeitlang "unnütze Bürger" zu sein, die am "Kampf mit äußeren Umständen « ${ }^{27}$ nicht teilnehmen, damit sich die Talente und die Freiheit des Einzelnen ganz entfalten können. Die Eigenzeit der Institution Universität ist somit gerade kein Trainingslager für die Kategorie der gegenwärtigen Nützlichkeit, son-

${ }^{26}$ Friedrich Schiller, Was heißt und zu welchem Ende studiert man Universalgeschichte? Eine akademische Antrittsrede, in: Ders., Sämtliche Werke, hg. von Gerhard Fricke/ Herbert G. Göpfert, Bd. 4: Historische Schriften, München 1980, S. 750.

27 Henrik Steffens, Über Idee der Universität, in: Ernst Anrich (Hg.), Die Idee der Universität. Die fünf Grundschriften aus der Zeit ihrer Neubegründung durch klassischen Idealismus und romantischen Realismus, 2., unveränd. reprograf. Nachdr. d. Ausg. Darmstadt 1956, Darmstadt 1964, S. 309-374, hier S. 324f. In dieser zweiten Vorlesung lässt Steffens die Gegner seiner Idee von der Universität zu Wort kommen, das heißt diejenigen, die die Universität auf die Teilnahme am gegenwärtigen Kampf um das Dasein des Staates und auf unmittelbare Nützlichkeit verpflichten wollen. Sie sagen etwa: »[...] der Staat aber unterliegt dem Drange der erscheinenden Gegenwart. Aus diesem Grunde hat der Staat recht, wenn er nur die Richtung des Geistes als ihm zugehörig anerkennt, die der Gegenwart untergeordnet ist und ihren Bedürfnissen entspricht." (ebd., S. 323f.) Und: "Zwar habt ihr versucht, das sogenannte Nützliche, welches nichts anders ist, als die Richtung der Tätigkeit eines jeden Bürgers zur Unterhaltung des Ganzen, zu schmähen und herabzuwürdigen. [...] Ihr habt unrecht. Nicht umsonst genießt ihr die Vorteile der Gesellschaft. Ein jeder muß seinen Platz im Staat bezahlen mit einer nützlichen Tätigkeit« (ebd., S. 325). 
dern, im Gegenteil, für die Transzendenz jeder Nützlichkeit, für die Freiheit zum Unnützen Wissen. Sie bildet, jenseits der Gegenwart, das Prinzip des Lebens des Staates. Aber genau wie für Rousseau ist auch für Steffens die Frage nach dem nützlichen bzw. nach dem Unnützen Wissen eine Frage der gesellschaftlich institutionalisierten Temporalität. Und genau wie bei Rousseau spielt auch für Steffens die Transzendenz der Gegenwart und die Reflexion der Zeitlichkeit eine zentrale Rolle:

Ihr behauptet, daß der Staat, gefangen von dem Drange der Gegenwart, bei allen Veranlassungen nur auf die Bedürfnisse derselben zu sehen hat. Aber was ist denn diese Gegenwart? Bestimmt durch die Vergangenheit, aufgelöst durch die Zukunft, läßt sie sich in keinem Momente fassen. Was ihr für die Gegenwart einrichtet, ist vergänglich wie sie. ${ }^{28}$

Bezieht man sich allein auf die Gegenwart, verliert man die Zukunft. Wer sich, so Steffens, dem "Drang der Umstände« unterwirft, der "vernichtet die lebendige Zukunft, eine kurze Gegenwart vorübergehend darzustellen«: ${ }^{29}$

Nur derjenige Staat, der es einsieht, daß das Entfaltende und Belebende in seiner Erscheinung nicht wiederum durch diese gemessen werden kann, daß es vielmehr, den unscheinbaren Keim der Zukunft enthaltend, als sein innerstes Heiligtum gepflegt werden muß, kann ohne Sorgen den Trübsalen der Zeit und dem unvermeidlichen Untergang bestehender Einrichtungen entgegensehen, denn was in ihm lebt, gehört nicht einer einzelnen Zeit oder bestimmten Umständen, vielmehr der ewig sich neu gestaltenden Geschichte. ${ }^{30}$

Mit seiner naturphilosophischen und organismustheoretisch begründeten Dialektik von Freiheit und Notwendigkeit, Leben und Staat, plädiert Steffens einerseits, wie Rousseau, für eine institutionelle Zeit, in der die Nützlichkeit der Gegenwart transzendiert wird und in der, gegen Rousseau, Zeit für Unnützes Wissen sein soll; andererseits begründet er diese Transzendenz aber selbst wieder mit einem, freilich ganz anders gelagerten, Rettungsnarrativ. Denn nur der Staat hat »die heitersten Hoffnungen für die Zukunft«, ${ }^{31}$ der auf diese Weise - und vor allem aufgrund des Studiums der Dichter der eigenen nationalen Vorzeit - sein inneres Leben erhält. Auch

28 Ebd., S. 341. Vgl. auch Friedrich Wilhelm Joseph Schelling, Vorlesungen über die Methode des akademischen Studiums, in: Ernst Anrich (Hg.), Die Idee der deutschen Universität, S. 19. Schelling wendet sich explizit gegen all jene, die »die Wissenschaft überhaupt nur als Nützlichkeit begreifen«.

29 Ebd., S. 342.

30 Ebd., S. 342 f.

31 Ebd., S. 363. 
Steffens bezieht in letzter Konsequenz die Einrichtung einer Eigenzeit für Wissen jenseits der Nützlichkeit auf das Überleben: Nicht als von der Gegenwart abgekoppelte Trainingszeit für die Fähigkeit, zukünftig rettungsnützliches Wissen zu erzeugen, sondern dialektisch, als Eigenzeit jenseits der Frage nach der Nützlichkeit, die als solche jene Freiheit ermöglicht, die für den Staat das innere Leben (und dadurch auch das äußere) und den Fortschritt allererst sichert.

VierzigJahre später kommt Robert Eduard Prutz in seinem Gesuch um eine Anstellung als Dozent der Literaturgeschichte an der Universität Halle auf dieses Rettungsnarrativ zurück. Zunächst räumt er ein, dass man immerhin fragen könne, wozu der Staat einen Dozenten einer Wissenschaft bezahlen solle, "die weitab liegt von den Interessen dieser Zeit, die keinen politischen Nutzen, keine praktische Wirksamkeit mehr hat «, ${ }^{32}$ um dann folgendermaßen zu antworten:

Was Preußen errettet hat aus einem noch viel tieferen Zusammensturz, als der gegenwärtige, nach dem Jahre 1806; was seine glorreiche Erhebung in den Freiheitskriegen ermöglicht und vorbereitet; was endlich ihm Kraft gegeben, jene Jahre des Irrthums und der Erniedrigung zu überdauern, ja was selbst auch sie nicht ohne einen gewissen Schimmer des Ruhms und der Größe gelassen hat - : die Macht der Bildung, die Stärke der Wissenschaft, die rettende Kraft des Geistes. ${ }^{33}$

An dieser Begründung, d.h. an der (wie auch immer vermittelten) Verwendung des Rettungsnarrativs und seines Bezugs auf das Leben und Überleben mag es liegen, dass das Modell Humboldt-Universität unserer Tage so widerstandslos durch die heute dominierenden Rettungsnarrative hat aufgelöst werden können. Durch jene ökonomisch-liberalistischen Diskurse, die uns permanent suggerieren, dass wir angesichts der Bedrohung durch die Globalisierung und den Wettbewerbsdruck auf dem Weltmarkt keine Zeit mehr für eine Zeit des unnützen, d.h. des nicht immer schon auf unmittelbaren Praxisbezug gemünzten Wissens haben. Denn an die Stelle jenes Zukunftsoptimismus und Fortschrittsglaubens ist in unserer »breiten Gegenwart« der prinzipielle Zweifel daran getreten, ob wir überhaupt eine $\mathrm{Zu}$ kunft haben, da sie, wenn überhaupt, nur mehr im Modus der Bedrohung erscheint: »Sie ist kein offener Horizont von Möglichkeiten mehr, sondern

32 Robert Prutz an das Ministerium: Gesuch um Anstellung an der Universität Halle, Berlin 13.8.1848, in: Uwe Meves (Hg.), Deutsche Philologie an den preußischen Universitäten im 19. Jahrhundert. Dokumente zum Institutionalisierungsprozess, Teilband I: Einführung, I. Teil: Universitäten, Berlin/New York 2011, S. 462-472, hier S. 467.

Ebd. 
eine Dimension, die sich zunehmend allen Prognosen verschließt und die zugleich als Bedrohung auf uns zuzukommen scheint." ${ }^{34}$

Rousseau hatte in einem ersten Schritt für eine Eigenzeit der Bildung plädiert, die sich von der Nützlichkeit der Gegenwart abkoppelt. Er hatte dann in einem zweiten Schritt diese Eigenzeit für ein Training der Suche nach nützlichem Wissen vorgesehen. Die Humboldt-Universität plädiert ebenfalls für die Institutionalisierung einer Eigenzeit für Bildung, allerdings als Ort, an dem das Wissen gerade nicht der Forderung der Nützlichkeit unterworfen sein soll. Begründet wird dies am Ende aber doch wieder mit einer Form von Nützlichkeit, nämlich mit der Nützlichkeit dieser Institution für das Leben und Überleben des Staats.

Unabhängig davon, ob das Wissen im Rahmen eines prinzipiellen $\mathrm{Zu}$ kunftsoptimismus und in Form institutioneller Eigenzeit (wie bei Rousseau und auch bei Steffens) oder aber gerade durch ihren Abbau (wie heute) an die Nützlichkeit gebunden wird, immer sorgen gerade Narrative der Rettung für den Begründungsrahmen und mit ihm für die besondere Evidenz der Nützlichkeit von Wissen. Diese Evidenz unterminiert und reduziert auch noch heute die ursprünglich viel weitere Bedeutung der Kategorie des Nutzens.

Der philosophische Begriff des Nutzens kreist ursprünglich um die Frage des Guten. ${ }^{35}$ Bis in die Neuzeit hinein steht das Nützliche in inklusiver Opposition zum Guten. Neben dem in sich selbst Guten (bonum honestum) und dem Lustvollen (bonum delectabile) - ist das Nützliche dessen dritte Form (bonum utile), d.h. ein Gut, das nicht um seiner selbst willen erstrebt wird. Zugleich aber, so Christian Thomasius, ist das Nützliche selbst ein Kriterium, um das wahre honestum vom falschen und das gute Lustvolle vom schädlichen zu unterscheiden. Dafür muss man vor allem gegenwärtiges und zukünftiges Gut trennen. So weist Thomasius die alte, aristotelische Unterscheidung der drei Formen des Guts zurück, insofern sie allein auf einen Unterschied im Zeitbezug beruhe:

Betrachte ich aber das Gute in Ansehen seiner selbst und seiner Gegenwärtigkeit / so heisset es ein belustigendes Gut. Endlich wenn ich seine Würckung betrachte / so heisset es nützlich / nemlich so ferne es ein neues Gute zuwegen bringet / oder das gegenwärtige continuiret. Und also ist kein anderer Unterschied unter dem nützli-

${ }^{34}$ Hans Ulrich Gumbrecht, Unsere breite Gegenwart. Aus dem Englischen von Frank Born, Berlin 2010, S. 17.

35 Vgl. Otfried Höffe, Nutzen, Nützlichkeit, in: Joachim Ritter (Hg.), Historisches Wörterbuch der Philosophie, Bd. 4, Basel 1984, Sp. 992-1008. 
chen und belustigenden Guten / als daß jenes auff zukünfftige Dinge / dieses aber auff gegenwärtige sein Absehen hat. ${ }^{36}$

Wenn demnach letztlich alles wahrhafte Gut nützlich ist, dann weil mit dem Leben und dessen Dauer ein Referenzrahmen gefunden ist, auf den jedes Gut im Hinblick auf seinen Nutzen bezogen werden muss. Thomasius stellt fest, »daß dasjenige alleine gut sey / was des Menschen Wesen und Kräffte am dauerhafftesten erhält / und vermehret / es möge diese Erhaltung und Vermehrung alsobald sich ereignen / oder erst eine geraume Zeit hernach zu spühren seyn«. ${ }^{37}$ Alles, was das Leben und das Wesen des Menschen erhält, ist gut und also nützlich. So sehr Thomasius auch gegen die scholastische Gelehrsamkeit und ihr Streben nach nutzlosem Wissen polemisiert, ${ }^{38}$ so sehr geht sein Begriff des Nutzens doch weit über die Sicherung des bloßen physischen Überlebens hinaus. Nutzen zielt für Thomasius auf die Glückseligkeit des Menschen, die letztlich in der Gemütsruhe und in der Selbsterkenntnis besteht. Seine Durchmusterung der Wissenschaften im Hinblick auf ihren Nutzen hat dann auch exakt in diesem Begriff sein entscheidendes Kriterium. Die bloß »belustigenden Studia «, ${ }^{39}$ wie die Historie, die Geographie oder die Mathematik und die "meisten Teile der Physic «, ${ }^{40}$ haben, so Thomasius, dennoch

ihren Nutzen darinnen / daß weil sie nicht eben grossen Kopffbrechens brauchen / und des Menschen natürliche curiosität in etwas vergnügen / sie zugleich unvermerckt denselben angewehnen stille zu sitzen und eine attention zu haben / welches zwey sehr nothwendige Stücke seyn die ernsthaffteren Studia zu befördern / und zu denenselben eine Lust zu erwecken. ${ }^{41}$

Man sieht, dass Thomasius gar nicht über die Nützlichkeit des Wissens spricht, sondern über den Nutzen, eine Wissenschaft zu betreiben. Belustigende Wissenschaften haben ihren Nutzen, insofern der Gelehrte sich an ihnen erquickt, »daß er folglich die ernsthafften Studia zu continuiren desto munterer wird. ${ }^{42}$ Die nützlichste Wissenschaft ist dann die, die »uns zur

36 Christian Thomasius, Einleitung zur Sittenlehre. Mit einem Vorwort von Werner Schneiders, Hildesheim 1968, S. 35.

37 Ebd., S. 12 f.

38 Vgl. Christian Thomasius, Ausübung der Vernunftlehre. Mit einem Vorwort von Werner Schneiders, Hildesheim 1968, S. 61ff.

39 Ebd., S. 56.

40 Ebd., S. 57.

41 Ebd., S. 57f.

42 Ebd., S. 59. 
Gemüths-Ruhe führet«, und das ist die "Sitten-Lehre ${ }^{43}{ }^{43}$ Der Begriff des Nutzens erhält hier also einen sehr weiten Umfang, nützlich ist jedes wahrhafte Gut, oder, wie es noch bei Adelung Ende des 18. Jahrhunderts heißt, "jede Verbesserung des Zustandes, sie sey von welcher Art sie wolle«. ${ }^{44}$ So kann man "Nutzen aus einer Lehre, aus einer Erkenntnis ziehen", jedenfalls dann, wenn man sie zur "Erweiterung seiner Erkenntniß oder zur Verbesserung des sittlichen Zustandes anwenden ${ }^{45}$ kann. Ähnlich umfassend und zunächst nicht auf den Körper und das bloße, physische Überleben bezogen, formuliert Jeremy Bentham: "Alles das ist dem Nutzen oder dem Interesse eines Individuums gemäß, was dahin zielt, die Totalsumme seines Wohlseins zu vermehren. ${ }^{46}$ Zwar basiert das Prinzip der Nützlichkeit bei Bentham und auch bei Claude-Adrien Helvétius auf dem antagonistischen Spiel von physischer Lust und physischem Schmerz, aber Bentham bezieht geistige Lust explizit mit ein: "[U]nter physischen Gütern und Uebeln verstehe ich aber die geistige Lust und Unlust ebensowohl als die sinnliche. « ${ }^{47}$ Wissen wäre nach dieser Definition auch dann nützlich, wenn es in physischer Hinsicht und zur Lebensrettung nutzlos, aber dennoch Medium geistiger Lust wäre. Wenn es jemanden geistig zufriedenstellt, zu wissen, was das Unnütze Wissen der Literatur ist, dann wäre das ein schon in diesem Sinne nützliches Wissen. Leider ist das aber nicht die derzeit herrschende Anschauung. Ihr gemäß muss das Wissen, das auf Kosten der Steuerzahler erhoben und gelehrt wird, sich auch durch einen Nutzen für diesen Steuerzahler rechtfertigen können - und dieser Nutzen ist dann eben nicht geistige Lust, zielt nicht auf die Sinndimension des Menschen, der die Welt, in der er lebt, verstehen will, sondern dieser Nutzen soll sich ökonomisch in verkaufbaren Produkten und Arbeitsplätzen niederschlagen. Statt zu fragen, ob wir ein Wissen wollen und ob es schöner und besser sei, ein bestimmtes Wissen zu haben, als es nicht zu haben, fragen wir ausschließlich danach, ob wir ein Wissen brauchen, d.h. nach dem ökonomischen Nutzen des Wissens.

Wo aber ist dieser ursprüngliche, umfassende Begriff des Nutzens geblieben, der Kategorien des Sinns und des geistigen Genusses mit einschließt? John Stuart Mill hatte 1861 noch einmal versucht, diesen weiten Begriff

43 Ebd., S. 55.

44 Johann Christian Adelung, Artikel Der Nutzen, Bd. 3, Sp. 547.

45 Ebd., Sp. 548.

46 Jeremy Bentham, Principien der Gesetzgebung, Köln 1833, S. 5.

47 Ebd., S. 6. Vgl. Manfred Kühn, War Hume Utilitarist?, in: Olaf Asbach (Hg.), Vom Nutzen des Staates. Staatsverständnisse des klassischen Utilitarismus: Hume, Bentham, Mill, Baden-Baden 2009, S. 92, sowie: Höffe, Nutzen, Nützlichkeit, Sp. 1004. 
von `utility` zu verteidigen. Mit seiner berühmten Formulierung »Es ist besser ein unzufriedener Mensch zu sein als ein zufriedenes Schwein «, ${ }^{48}$ zeigte er explizit diese Differenz. Und er wies auf die Bedingungen hin, die es braucht, damit es überhaupt sogenannte shöhere Bedürfnisser geben kann, und diese Bedingungen sind im Wesentlichen eine Frage der Zeit: »Die höheren Bedürfnisse und geistigen Interessen der Menschen sterben ab, weil es ihnen an Zeit und Gelegenheit fehlt, sie zu pflegen. «"

Der Begriff des Nutzens hatte, wie umfassend er auch zunächst gedacht worden war, dennoch die Tendenz, sich selbst zu spalten, in den scheinbaren und den wahren Nutzen, den subjektiven und den objektiven, den quantitativen und den qualitativen etc. Der Zukunftsbezug des wahren Nutzens im Gegensatz zum bloß scheinbaren Augenblicksnutzen, wie ihn schon Thomasius formuliert und wie er dann, vor allem ausgehend von Benthams Mathematisierung der Folgenabschätzung, gedacht wird ${ }^{50}$ ist ein Nutzen, der aufgrund seines quantitativen Kalkulationscharakters jede unmittelbare Evidenz verliert. Sucht man aber die Evidenz des Nützlichen, so sind es gerade Narrative der Rettung, Situationen auf Leben und Tod, die das Nützliche als Nützliches für das blo/se Leben besonders eindrücklich und unbestreitbar in Szene setzen. Dieses Rettungsnützliche ist dann zwar evident nützlich, aber zugleich auf das physische Leben und seine unmittelbare Rettung beschränkt. Hier wird die Zukunft allein in der Gegenwart gewonnen, für eine Einrichtung einer zukunftsbezogenen Eigenzeit ist dann keine Zeit mehr.

Die Reduktion der Kategorie des Nutzens auf die letztlich physischen oder ökonomischen Aspekte im Hinblick auf die Nützlichkeit des Wissens erfolgt durch das herrschende politische und ökonomische Paradigma der Rettung und seiner spezifischen Temporalität. Je weniger man glaubt, dass auch in Zukunft noch Zeit sein wird, weil man in der Gegenwart die Zukunft retten muss, desto weniger glaubt man, sich leisten zu können, eine Zeit zu institutionalisieren, in der man Zeit verliert. Wer auf der Flucht ist, um sich zu retten, hat in der Tat keine Zeit zu verlieren. Und so werden die Bedürfnisse der Gegenwart, der unmittelbare Nutzen allen Wissens zum entscheidenden Kriterium für das, was und wie gelernt werden soll. Die Nicht-Schulzeit, die Schulzeit und die Universitätszeit werden verkürzt ${ }^{51}$ die Universität wird

${ }^{48}$ John Stuart Mill, Utilitarianism. Der Utilitarismus. Englisch. Deutsch, übers. von Dieter Birnbacher (Hg.), Stuttgart 2010, S. 33.

49 Ebd., S. 35.

50 Vgl. Jeremy Bentham, Principien, S. 43-45.

51 "Das deutsche Bildungssystem raubt den Jugendlichen im europäischen Vergleich wertvolle Zeit, die sie für Familiengründung, Beruf und den Aufbau ihrer Altersversorgung 
zur Agentur für Praxisbezüge, gelernt werden soll nur noch, was wenig später (oder schon gleichzeitig im Praktikum) auch tatsächlich gebraucht wird, für eine Zeit nutzloser Gegenwart ist keine Zeit mehr. Dass unter solchen Umständen insbesondere das Wissen der Kultur- und Literaturwissenschaft als tendenziell unnütz erscheint, ist klar, denn das organismustheoretische Argument von der Dichtung als Paradigma des inneren Lebens des Staates oder auch der "nationale[n] Wichtigkeit«, ${ }^{52}$ wie es die Entstehung der Literaturwissenschaft im 19. Jahrhundert begleitet und eine explizite und aufwendige Begründung seiner Nützlichkeit entbehrlich gemacht hat ${ }^{53}$ steht nicht mehr zur Verfügung. Auf diese Situation nun mit Versuchen zu antworten, die Nützlichkeit des Wissens der Literaturwissenschaft anderweitig zu begründen, würde dabei vermutlich nur die Kategorie der Nützlichkeit

nutzen könnten. Wenn deutsche Akademiker im Durchschnitt erst mit 28 Jahren ins Berufsleben eintreten, ist das eine Vergeudung von Ressourcen für die Sozialversicherungssysteme und letztendlich für das gesamte Gemeinwesen. Deshalb muss gelten: früher in die Schule und früher in den Beruf." (Regierungserklärung des Bayerischen Ministerpräsidenten Dr. Edmund Stoiber am 6. November 2003, abrufbar unter: http://www.gdv-bayern. de/2006/01aktuelles/pdf/RegErkl.pdf) [Datum des letzten Zugriffs: 20.08.2015]. Die überhastete Einführung des verkürzten Gymnasiums (G 8) war Folge des sogenannten Pisa-Schocks. Erstaunlich ist im Rückblick, dass eine wirkliche Begründung gar nicht gegeben wurde, es reichte der Hinweis darauf, dass andere schneller sind und dass das im globalen Wettbewerb ein Nachteil sein könnte: »Eines der wichtigen Ergebnisse von PISA ist, dass es in Deutschland im internationalen Vergleich einen rgroßzügigen Umgang mit Lebenszeit gibt, wie es dort in einer freundlich gehaltenen Formulierung von Professor Baumert heißt." (Rede der Ministerin für Schule, Jugend und Kinder NRW Ute Schäfer zum Thema 'Aktuelle schulpolitische Perspektiven unter besonderer Berücksichtigung des Gymnasiums bei der Jahresversammlung der Westfälischen Direktorenkonferenz am 24.11.2003, abrufbar unter: http://westfaelische-direktorenvereinigung.de/ wordpress/wp-content/uploads/2012/11/Rede-schaefer-24-11-2003.pdf) [Datum des letzten Zugriffs: 20.08.2015]. Einen solchen "großzügigen Umgang mit Lebenszeit« kann man sich angesichts der Konkurrenz mit Chinesen oder Koreanern nicht mehr leisten, wenn man im globalen Wettbewerb nicht untergehen möchte. Man muss dann den drohenden Untergang gar nicht an die Wand malen, es reicht, von »besseren Chancen« zu reden: "Unsere Jugendlichen sollen die bestmögliche Ausgangsposition für ihren Start in das Leben haben. Sie sollen hervorragend ausgebildet werden. Aber sie sollen auch mit Jugendlichen aus anderen Ländern mithalten können, die früher in das Berufsleben einsteigen und damit in unserer globalen Welt bessere Chancen haben. Deshalb werden wir das Gymnasium auf acht fahre verkürzen." (Regierungserklärung des Bayerischen Ministerpräsidenten Dr. Edmund Stoiber am 6. November 2003).

52 Klaus Weimar, Geschichte der deutschen Literaturwissenschaft bis zum Ende des 19.Jahrhunderts, München 1989, S. 247.

53 Weimar zitiert den österreichischen Minister Leo Graf Thun-Hohenstein (1858): "Das unabweisbare Bedürfnis des Bestandes einer bleibenden Lehrkanzel für deutsche Sprache und Literatur an jeder Hochschule des Kaiserreichs bedarf wohl keiner näheren Begründung.« Ebd. 
selbst (und ihre Tendenz zum Testfall der Rettungsnützlichkeit) affirmieren. Sicher ist es richtig, darauf hinzuweisen, dass zum Wissen der Literaturwissenschaft auch ein »kommunikatives Wissen gehört«, nämlich das Wissen, wie man Wissen kommuniziert, ${ }^{54}$ aber das wiederum betrifft nur einen Teil des Wissens der Literaturwissenschaft.

Nötig scheint mir demgegenüber eine Zurückweisung des Bezugs des Wissens auf Nützlichkeit überhaupt und eine Zurückweisung des regierenden Rettungsnarrativs. Wer auf der Flucht ist, nimmt vermutlich wirklich eher einen Laib Brot mit als eine Werkausgabe von Kafka, aber wir sind nicht auf der Flucht. Es ist eben dieses Rettungsparadigma, das Rousseau seiner Pädagogik der Nützlichkeit zugrunde gelegt hatte, das den Begriff des Nutzens aufspaltet und ihn an das physische Überleben bindet. In Bezug auf das Wissen sollte aber, außerhalb des Ausnahmezustandes der Rettung, nie die Frage sein, ob ein Wissen nützlich ist, sondern schlicht, ob ein Wissen gewünscht wird. Erstaunlicherweise muss ja naturwissenschaftliche Forschung selbst da, wo sie keinen ökonomischen Nutzen abwirft (z.B. Grundlagenforschung), keinen Rechtfertigungsdiskurs führen - und wirklich ist ja nie die Frage, ob wir das Wissen brauchen, dass Pluto gar kein Planet ist oder dass Hummeln die einzigen Insekten sind, die rückwärts fliegen können - die Frage ist immer nur, ob wir in einer Welt leben wollen, in der es unter anderem auch dieses Wissen gibt. Rousseaus Pädagogik eines Trainingslagers des nützlichen Wissens zeigt ja gerade, wie sehr die umfassende und eben nicht auf das Nützliche bezogene Neugier des Kindes zugerichtet und beschnitten werden muss, wie viel disziplinatorischer Aufwand nötig ist, um die theoretische Neugier auf die Suche nach dem Nützlichen zu reduzieren. Es gibt aber sehr viele Gründe dafür, etwas wissen zu wollen, das Spektrum reicht von der Lebensrettung (Rumpelstilzchen!) ${ }^{55}$ bis zur Befriedigung bloßer Neugier und dem Wunsch, sich in der Welt durch Wissen zu beheimaten. Bei der gesellschaftlich nötigen Aushandlung über die Ausschöpfung dieses Spektrums sollte dieser Grund nicht ganz unter die Räder kommen.

54 Vgl. Heinrich Bosse, Schul- und Brotwissenschaft. In: Merkur 68/3 (2014), S. 221-231.

55 „Endlich sagte es: in drei Tagen komm ich wieder und hole das Kind, wenn du aber dann meinen Namen weißt, so sollst du das Kind behalten!« - Kinder- und Haus-Märchen, gesammelt durch die Brüder Grimm, Berlin 1812/15, S. 254. 
